Gut, 1969, 10, 121-130

\title{
Experimental study of devascularization of the colon
}

\author{
ADRIAN MARSTON, R. W. MARCUSON, M. CHAPMAN, AND J. F. ARTHUR \\ From the Department of Surgical Studies, The W. H. Collins Department of X-ray Diagnosis and The Bland- \\ Sutton Institute of Pathology, The Middlesex Hospital, London
}

Recent clinical experience (Boley, Schwartz, Lash, and Sternhill, 1963; McGovern and Goulston, 1965; Marston, Pheils, Thomas, and Morson, 1966; Miller and Knox, 1966; Sturdy, 1968) has suggested that many cases of hitherto obscure colonic disease may in fact originate from vascular insufficiency. Some of these cases are directly attributable to blockage of a major vessel by thrombosis or embolism (Marston et al, 1966; Miller and Knox, 1966) whilst in others the ischaemia originates from occlusion or spasm of the smaller intramural vessels (Boley, Krieger, Schultz, Robinson, Siew, Allen, and Schwartz, 1965). The diagnosis of colonic infarction is not always easy and the clinical features, $x$-ray appearances, and pathology are still not completely defined. The purpose of the present work has been to produce a number of standardized controlled vascular occlusions in the experimental animal and to study their general and local effects in the hope of increasing our knowledge of what may be expected in the clinical situation.

Dogs were selected for the investigation for reasons of availability and ease of handling, and also because there is considerable laboratory experience in the behaviour of the ischaemic intestine in this species (Marston, 1962). The pattern of arterial supply to the dog's colon is shown in Figure 1. Blood arrives through the common colic artery, a branch of the superior mesenteric trunk, and the caudal (inferior) mesenteric artery. The common colic artery divides into the middle colic artery, which is distributed much as in the human colon, and the ileo-caeco-colic artery, which runs down the ascending colon and divides into two vessels which finally inosculate with the termination of the superior mesenteric. The caudal mesenteric artery runs straight to the colon and forms a 'T-junction', which above connects with the left branch of the middle colic, and below continues to form the superior rectal artery. In this way a continuous vessel, running along the inner side of the colonic loop, is formed: the marginal artery of the colon.

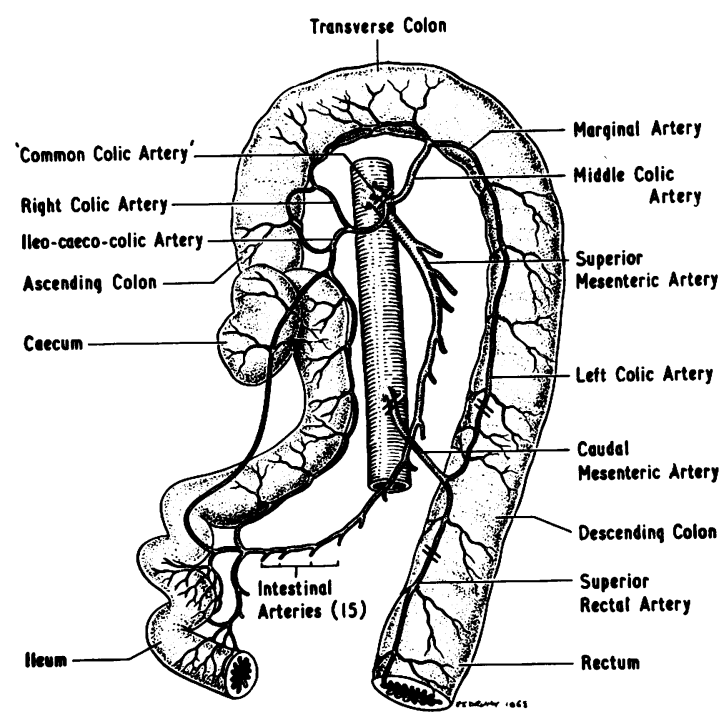

FIG. 1. Anatomy of the blood supply to the dog's colon, showing points of ligature.

\section{METHODS}

All dogs were as far as possible admitted to the laboratory population at least one week before the proposed surgical procedure. Baseline determinations were made of weight, total white cell count, serum alkaline phosphatase, serum glutamic oxalate transaminase (SGOT), serum glutamic pyruvate transaminase (SGPT), and lactic dehydrogenase (LDH). The dog was then assigned to a particular group and a devascularization operation performed (Fig. 2).

SURGICAL PROCEDURES Anaesthesia was induced with pentobarbitone $30 \mathrm{mg}$ per $\mathrm{kg}$ and supplemented by small additional doses as necessary. A cuffed endotracheal tube was passed. The abdomen was entered through a midline incision under sterile conditions and the normality of the peritoneal contents verified. Animals with obvious intraabdominal disease were excluded from the series. In nine dogs (groups A, B, and C) a silk ligature was applied to 


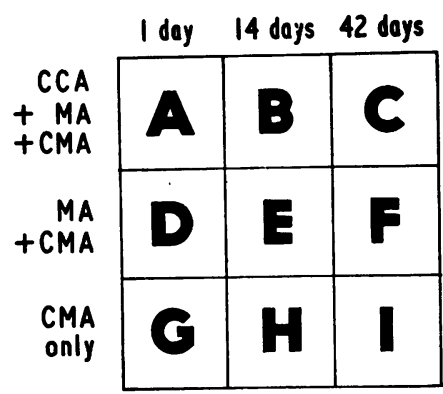

Three dogs in each group, plus six controls anaesthesia without prior bowel preparation. A Foley catheter was introduced into the rectum and the balloon $(50 \mathrm{ml})$ was inflated and withdrawn against the anal sphincter. A mixture of equal parts of Micropaque (Damancy) and water was introduced through the catheter until the caecum was reached. The dogs were screened in the supine position with the right side raised using a Siemans mobile image intensifier (screening factors 0.5 MA, $57 \mathrm{KV}$ ). A series of three films was then taken (1) with the large bowel filled with barium; (2) after draining the excess barium via the catheter; and (3) after air insufflation to obtain an air-contrast film (exposure factors $26 \mathrm{MA}, 57 \mathrm{KV}, 0.2 \mathrm{sec}$ ) using Blue Brand standard Kodak films and high speed intensifying screens.

\section{RESULTS}

CLINICAL FEATURES In general the animals withstood the operative procedure very well and were eating and drinking normally on the first postoperative day. Not unexpectedly those subjected to ligation of all three vessels, referred to as 'subtotal devascularization' (groups A, B, and C), and to ligation of the caudal mesenteric and marginal arteries (groups D, E, and F) were considerably more affected than those in which the caudal mesenteric artery only had been ligated. One death from necrosis of the bowel and peritonitis occurred on the fourth postoperative day after ligation of the caudal mesenteric and marginal arteries. Diarrhoea was observed in 10 animals and fresh blood in two.

SIGMOIDOSCOPY The normal sigmoidoscopic appearances were learned from preoperative examinations. Thereafter, all dogs were routinely sigmoidoscoped, without preparation, at one, 14, and 42 days, that is, immediately before the barium enema, except in a few early experiments, or where the animal was to be sacrificed after the radiograph.

The most striking features were seen at one day in the major ischaemia group. The bowel was empty. In three dogs a circumferential mucosal slough, 12 to $19 \mathrm{~cm}$ long, had occurred with congested, purplish mucosa above and below it.

At 14 days small discrete ulcers were seen in two dogs, both of which had had some lesions on the first postoperative day. Both on sigmoidoscopy and in the excised specimen, the distribution of the ulcers tended to be linear, as noticed by Boley et al (1965).

At 42 days one definite stricture was seen, at $13 \mathrm{~cm}$. It was about $4 \mathrm{~cm}$ long, smooth, rigid and indistensible. Attempts to pass the instrument through it caused the mucosa to bleed. Less severe lesions were frequently seen and consisted of mucosal oedema, spasm, and haemorrhage into submucous lymphoid nodules. Ligation of the caudal mesenteric artery 


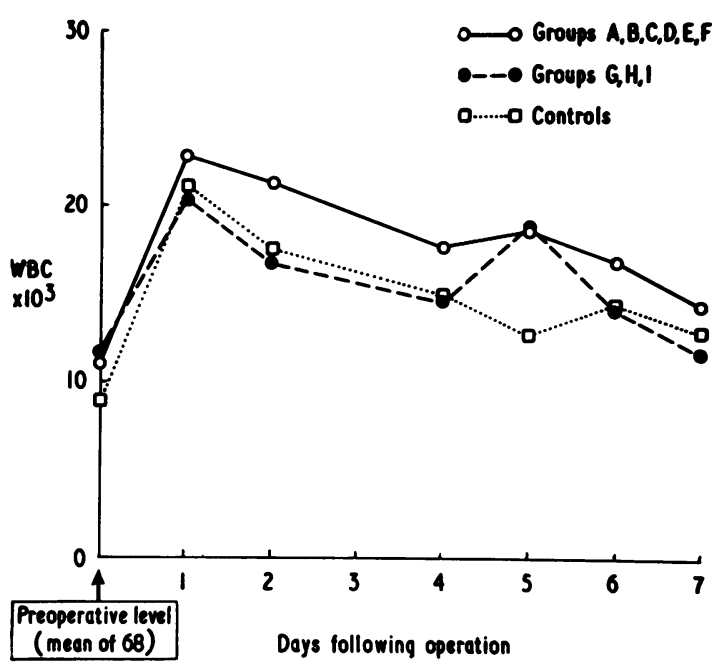

FIG. 3. Changes in leucocyte count following operation.

alone produced minor abnormalities in one animal out of six, the remaining five being unaffected.

LEUCOCYTE COUNTS The mean preoperative leucocyte count for the dogs as a whole was 10.700 \pm $3 \cdot 700$. Following devascularization there was a steep rise in the mean count to $22 \cdot 300 \pm 6.600$, which tended to return to normal by the end of the first

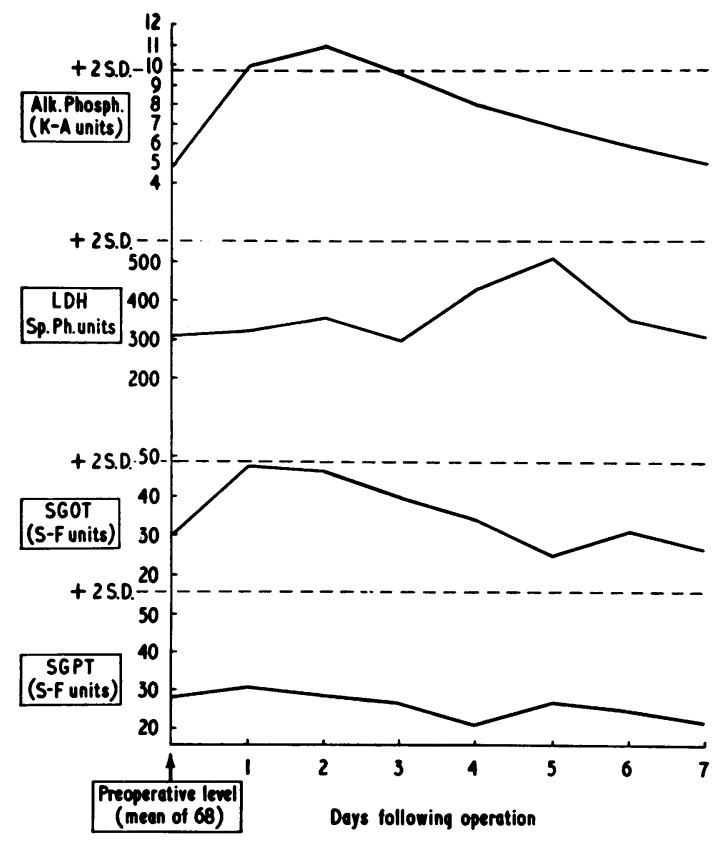

FIG. 4. Changes in serum enzyme levels following operation. postoperative week. This leucocytosis was not significantly different in the cases of major ischaemia (see Fig. 3).

CHANGES IN SERUM ENZYMES The preoperative levels of all the enzymes agree well with the values for normal dogs quoted by Bedrak (1965). Repeated preoperative estimations in our experimental group of dogs showed that in any one animal the alkaline phosphatase level remained relatively constant but that there was quite marked variation between individuals. This variability was not seen with the other enzymes.

The changes in mean serum enzyme levels for the



FIG. 5. Changes in alkaline phosphatase levels with varying degrees of ischaemia.

whole series, over the first seven days following devascularization, are summarized in Figure 4. There was no change in the level of SGPT. The SGOT showed an early rise which barely reached a significant level (two standard deviations above the baseline), and the LDH level rose slightly towards the end of the week. There was, however, a steep and significant rise in alkaline phosphatase levels during the first few days after operation, which was related to the degree of vascular injury (see Fig. 5). The greatest increase appeared in the major ischaemia group, though smaller rises are found in the minor ischaemic and sham groups. The apparent relationship of this rise to the degree of ischaemia is discussed below. No such difference between the groups exists for LDH and SGOT. 
RADIOLOGICAL FINDINGS The dog's colon lacks haustral folds and is uniformly tubular. The mucosa appears featureless. The distal portion of the colon is situated on the left side of the abdomen and there is no recognizable sigmoid loop. In the upper abdomen there is a flexure and the more proximal portion of the colon is situated medially. A short distance from this flexure the large bowel ends in a finger-like caecum. These appearances (Fig. 6) are constant and for this reason preoperative investigations on normal dogs were omitted after the first few experiments. Postoperative enemas were routinely performed at one, 14 , and 42 days according to the type of experiment. The abnormal features noted postoperatively were an increased incidence of spasm, mucosal oedema or 'thumbprinting', and stricture formation.

Spasm was defined as a segment of narrowing present on the first and second films, which was abolished by air insufflation. It was found constantly in the more severe vascular injuries at 14 days, but never in the controls.

Mucosal oedema produced scalloping of the margins of the barium column on the filled films. In the film taken after draining the excess barium, coarse mucosal folds were demonstrated in the affected segment, which was always at about the level of the iliac crest. Mucosal oedema was also well demonstrated in the air contrast films (Fig. 7). This finding was demonstrated in the one-day films of three dogs submitted to ligation of the caudal mesenteric and marginal arteries and of six dogs following subtotal devascularization. It was not found in any experiment involving ligation of the caudal mesenteric artery alone, or in any 14- or 42-day films.

A stricture was considered to be present only when a constant narrowing remained unaltered at all three films of the series (Fig. 8). This was found in one animal, which also demonstrated pathological changes (see below).

PATHOLOGICAL APPEARANCES The fixed specimens were examined macroscopically for mucosal ulceration, strictures, changes in thickness of the bowel wall, and peritoneal reactions. Blocks were taken from all ulcers and strictures and also from apparently normal areas in each specimen.

The histological material was prepared by standard methods (Clayden, 1962) and sections were stained with haematoxylin and eosin, by Van Gieson's method, and by Perl's Prussian blue method to demonstrate haemosiderin. The principal lesions found were small mucosal ulcers, extensive necrotic ulcers, and strictures of the bowel.

Small mucosal ulcers Clearly defined areas of mucosalulceration, 2 to $3 \mathrm{~mm}$ diameter, usually with a haemorrhagic base and sometimes surrounded by smooth atrophic mucosa, tended to occur in a longitudinal linear distribution in the affected segment of bowel. The remainder of the bowel wall appeared normal. Such lesions were seen in four animals with occlusion of the caudal mesenteric artery only, in four with occlusion of the caudal mesenteric and marginal arteries, and in two with subtotal devascularization.

Microscopically there was loss of mucosa over a small area (usually less than $1 \mathrm{~mm}$ diameter). The floor of the ulcer was formed of inflammatory

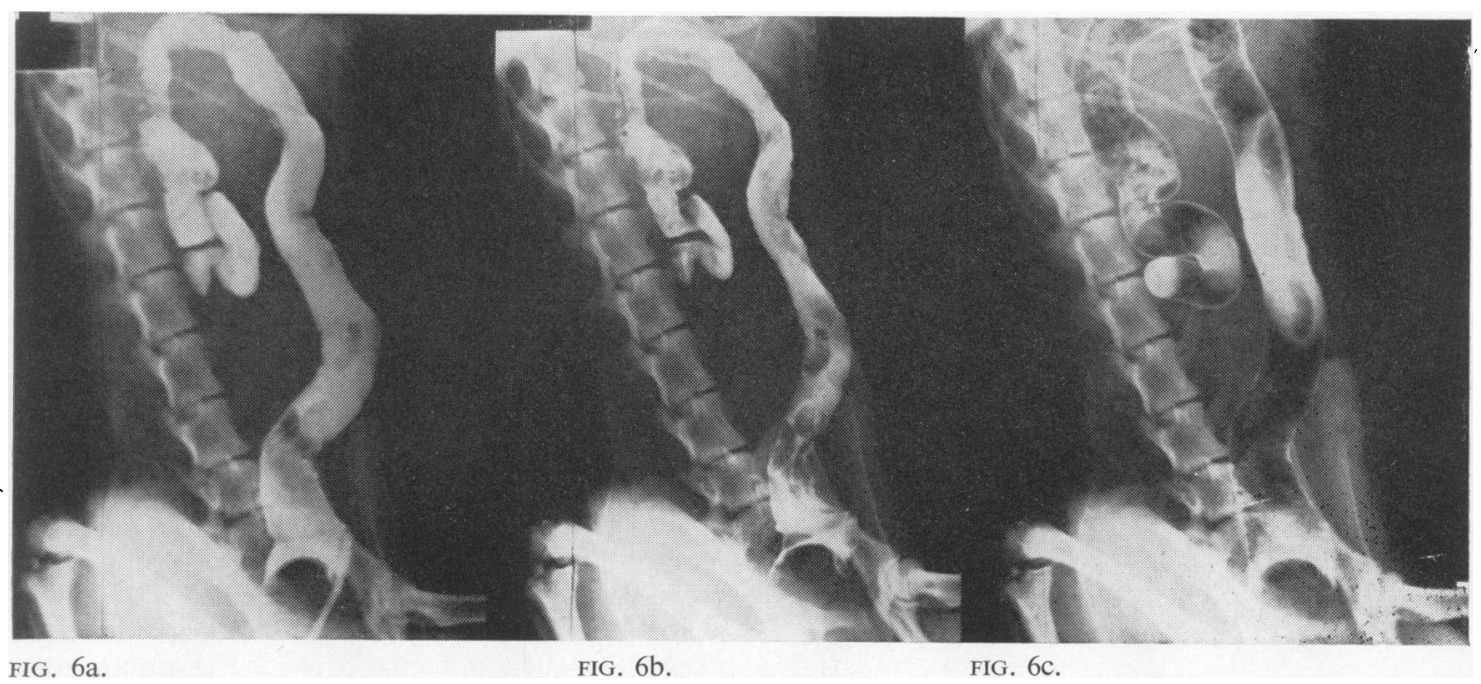

FIG. 6. Normal barium enema appearances: a colon filled with barium; b after drainage; c air-contrast film. 


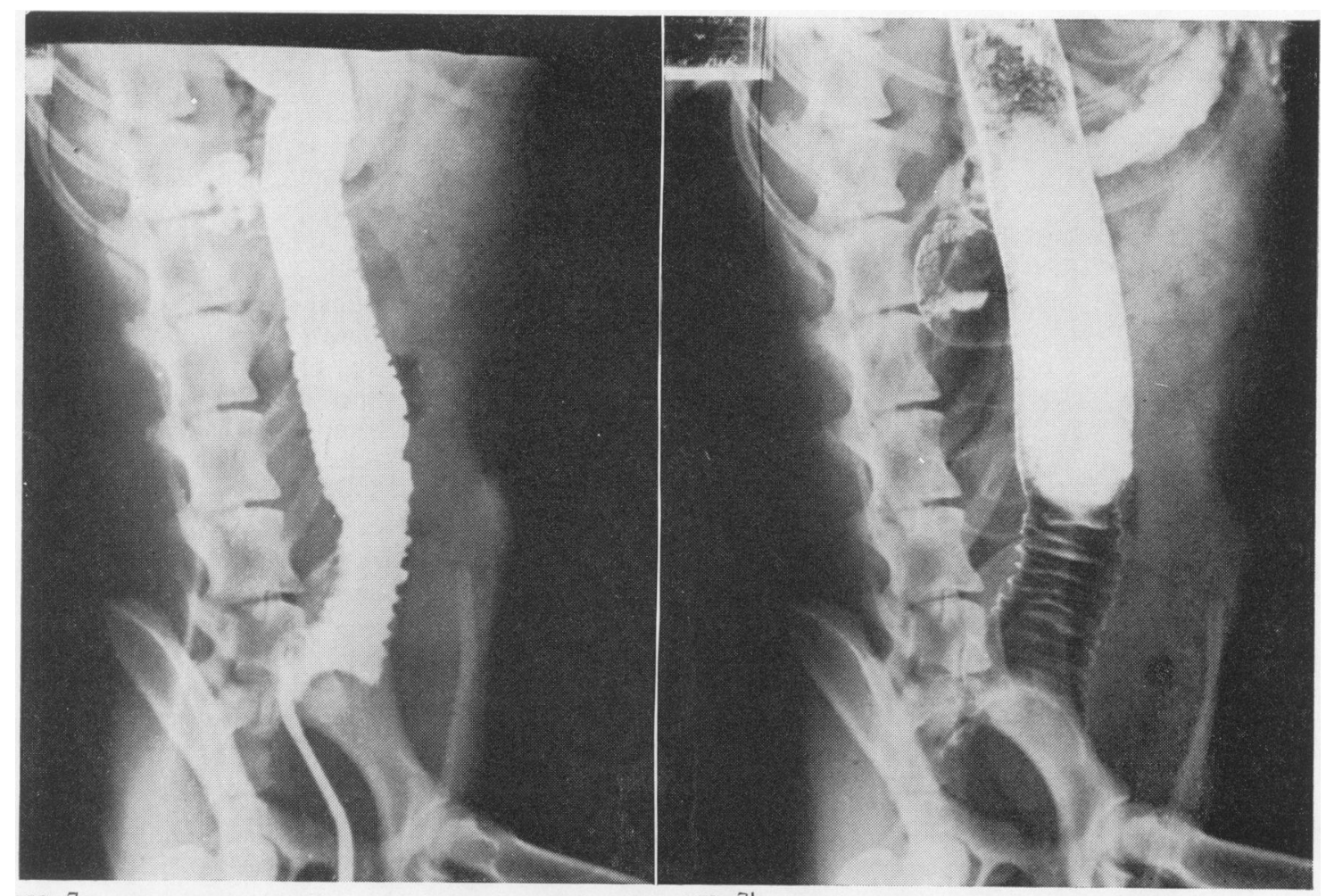

FIG. 7a.

FIG. $7 \mathrm{~b}$

FIG. 7. Barium enema one day following ligation of the caudal mesenteric and marginal arteries showing 'thumb printing' due to mucosal oedema: a filled film and $\mathrm{b}$ air-contrast.

FIG. 8. Barium enema 42 days following ligation of the caudal mesenteric and marginal arteries showing a persistent stricture.

FIG. 8 . 


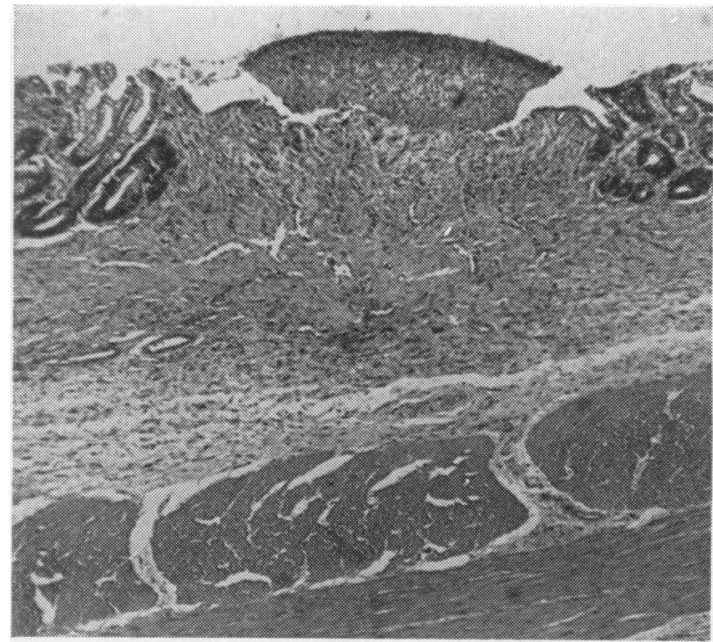

FIG. 9. Ulceration of the mucosa 14 days after ligation of the caudal mesenteric artery $(H$ and $E \times 30)$. granulation tissue. There was oedema of the submucosa with some increase in vascularity and a moderate inflammatory infiltration of non-specific type. In four specimens the mucosa round the ulcers was thin and atrophic, in the remainder it was normal. Four of the 10 specimens showed free iron in macrophages in the submucosa and three showed free iron in the peritoneal layer. Four specimens showed thickening of the muscle layer, the muscle fibres showed no degenerative changes, and there was no significant fibrosis. This appearance was thought to indicate muscle shortening due to spasm. Five specimens showed inflammatory changes in the peritoneal layer (Fig. 9).

Extensive necrotic ulcers In more severe ischaemia irregular areas of ulceration covered with adherent slough were seen, usually involving the whole circumference of the bowel over a length of 3 to $9 \mathrm{~cm}$. The edges of the ulcers showed clear demarcation from the surrounding normal mucosa. There was
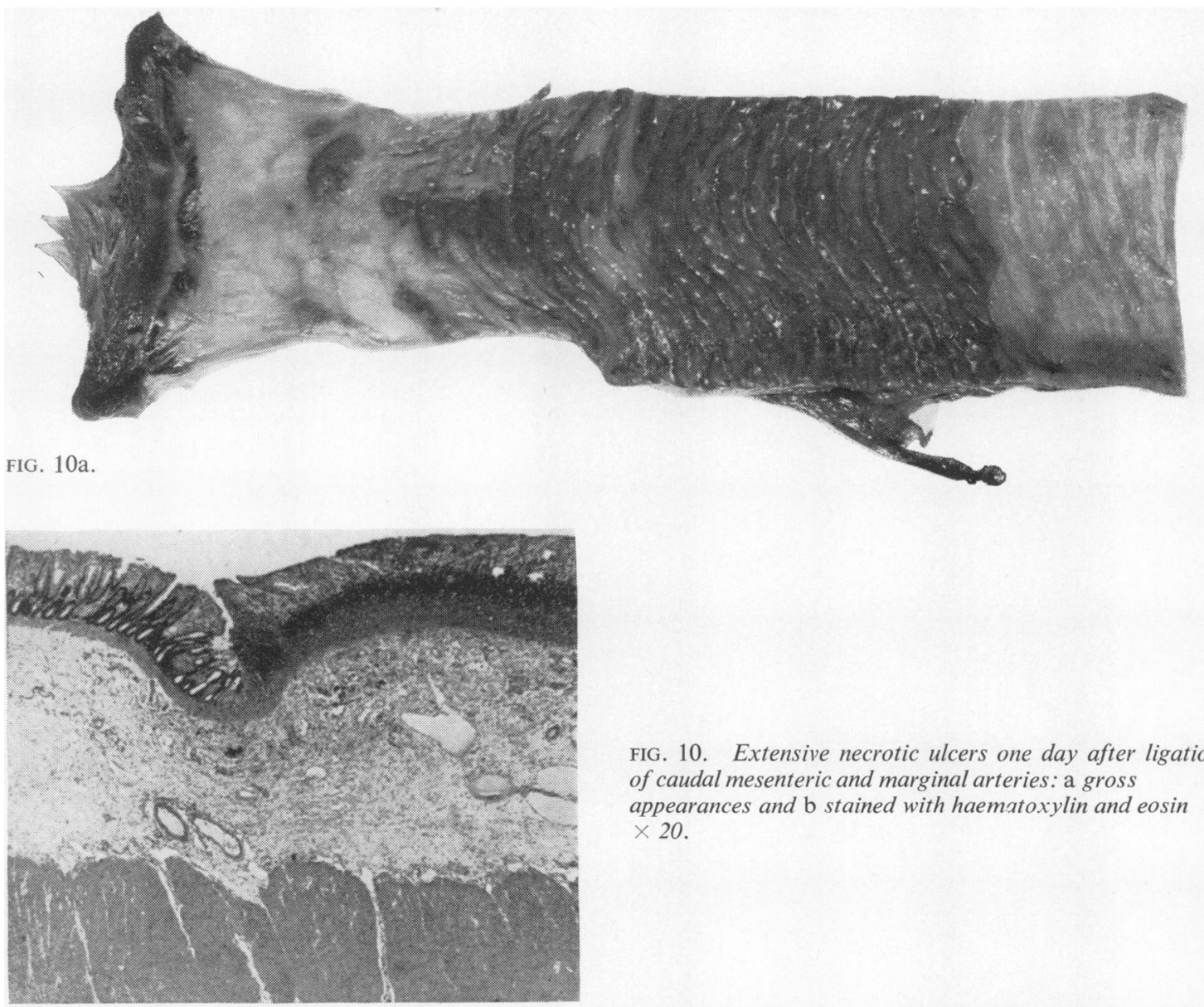

FIG. 10. Extensive necrotic ulcers one day after ligation of caudal mesenteric and marginal arteries: a gross appearances and $\mathrm{b}$ stained with haematoxylin and eosin $\times 20$.

FIG. $10 \mathrm{~b}$. 




FIG. 11a.

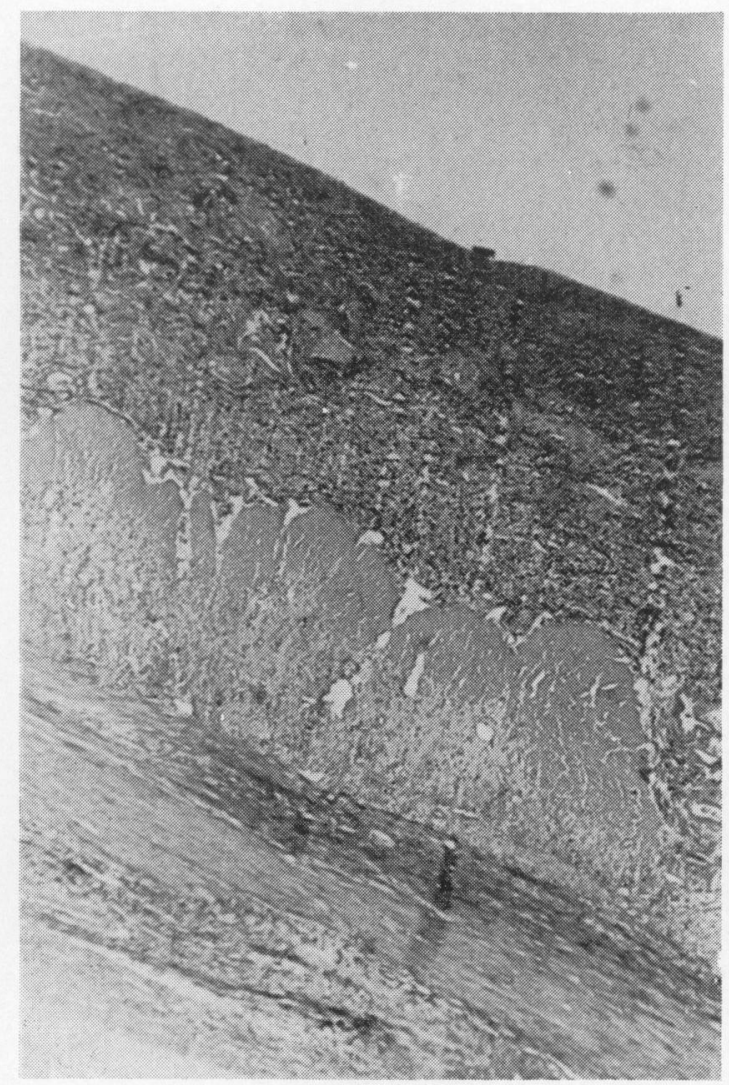

FIG. $11 b$.

FIG. 11. Stricture appearing 14 days after subtotal devascularization: a gross appzarances and b stained with haemotoxylin and eosin $\times 40$. slight thickening of the bowel wall in the ulcerated area with congestion of the peritoneal surface and in some cases fibrinous exudate on the peritoneum. These lesions were not seen in any of the animals with occlusion of the caudal mesenteric artery only, but were present in two animals with occlusion of the caudal mesenteric and marginal arteries and three with subtotal devascularization (Fig. 10).

Microscopically these showed large areas of total necrosis of the mucosa. The edges of these areas were very sharply defined and the mucosa round them was normal. Normal submucosa was replaced in the ulcerated area by a vascular granulation tissue containing many fibroblasts and a sparse inflammatory infiltrate, mainly consisting of lymphocytes and plasma cells with only a few polymorphonuclear leucocytes. Free iron was seen in macrophages in one specimen only. In the muscle layers two specimens showed considerable muscle fibre degeneration and one showed some fibrosis. Four specimens showed some peritonitis.

Strictures of the bowel Reduction of the circumference of the bowel by $1 \mathrm{~cm}$ or more was regarded as a stricture. The colon wall was approximately twice its normal thickness in a strictured area. The lengths of the strictures varied from $2.5 \mathrm{~cm}$ to $9 \mathrm{~cm}$. The mucosa in all cases showed areas of ulceration, usually small. The remaining mucosa in the strictures was smooth and appeared atrophic. Strictures were seen in one animal with occlusion of the caudal mesenteric artery, in three with occlusion of the caudal mesenteric and marginal arteries, and in one with subtotal devascularization (Fig. 11).

Microscopically four specimens showed thickening of the muscle layer with no abnormality of the muscle cells and no increase in muscle interstitial tissues. This appearance was thought to be due to muscle shortening.

One specimen showed fibrosis of the muscle and 
three showed increased fibrosis in the submucous layer.

Four had small mucosal ulcers with atrophic mucosa around them and one showed extensive ulceration.

Four showed free iron in macrophages either in the submucosa or peritoneum.

\section{DISCUSSION}

Three groups of workers have previously investigated the effects of colonic devascularization in the experimental animal. Boley et al (1963) studied five cases of ischaemic colitis in human subjects and were able to reproduce the appearances by ligating the colonic arteries in a number of dogs. Later Boley et al (1965) extended these experiments and altered their technique by using injections of glass microspheres into the arteries supplying an isolated segment of colon. No histological studies have been reported by these workers. Hukuhara, Kotani, and Sato (1961) and de Villiers (1966) have been interested more specifically in the state of the ganglion cells following a period of ischaemia and have used a technique in which an isolated segment of colon was perfused with Tyrode solution for four hours and the blood supply then restored. Our interest has been in the structural effects of a pure ischaemic lesion of the colon as occur clinically from embolus or thrombosis of aortic branches, without the added factor of foreign bodies in the minute vessels or abnormal pericellular electrolyte concentrations, and for this reason we have reverted to the technique of simple arterial ligation. By varying the site of ligature and the age of the lesion studied, we have been able to gain some information regarding the natural history of this condition in the experimental animal.

Devascularization of the colon, even when all named routes of blood supply have been obliterated, is a procedure which is withstood well by the healthy dog. Only one death occurred in the series and gross systemic upset and melaena were unusual.

The leucocyte count rose steeply on the day following operation, and gradually subsided to the normal level by the end of the first week, which is reminiscent of the effects of acute intestinal ischaemia in the human patient, where peripheral leucocytosis may precede physical signs. This was, however, a non-specific effect as there was no relation between the extent of leucocytosis and the severity of the ischaemia, and a rise in white cells was also seen in animals which had undergone a 'sham' operation.

Other workers (Dagher, Panossian, and Saab, 1967; Rudolph, Schaefer, Dutton, and Lyons, 1957) have postulated rises in serum transaminase activity in intestinal ischaemia. However, in our series the elevation of SGOT and LDH (Figure 4) levels all appear to be non-specific, occurring to a similar extent in the ischaemic and sham groups. The changes noted are probably due to the operative procedure. The rise noted in serum alkaline phosphatase, however, is of a different nature. There was a significant $(0.05>P>0.01)$ difference between the levels recorded in those animals undergoing a major devascularization operation on the one hand, and in the control groups on the other, which is most marked on the second postoperative day.

Because of the peculiar distribution of normal preoperative levels of alkaline phosphatase in the dog the rise in enzyme level was thought to give a better measure of change than the actual level reached. Analysis of major ischaemia and sham groups with these figures did not, however, appreciably alter the significance of the difference. There was, furthermore, a close relationship between the degree of colonic damage produced (as opposed to the vascular lesion inflicted) and the postoperative rise in alkaline phosphatase, as is shown in the following Table.

\section{TABLE}

\begin{tabular}{llll} 
Group & $\begin{array}{l}\text { Colonic } \\
\text { Damage }\end{array}$ & $\begin{array}{l}\text { Mean Rise in Alkaline } \\
\text { Phosphatase on First Post- } \\
\text { operative Day (K-A units) }\end{array}$ \\
\hline A-F & Extensive ulcers & (7 dogs) & $9 \cdot 8$ \\
& Small ulcers & (4 dogs) & $2 \cdot 9$ \\
G-I & No change & (5 dogs) & $5 \cdot 0$ \\
& Small ulcers & (1 dog) & $1 \cdot 7$ \\
& No change & (5 dogs) & $2 \cdot 2$ \\
& Controls & (5 dogs) & 3.5
\end{tabular}

This rise in alkaline phosphatase, apparently specifically related to colonic ischaemia, was of great interest and prompted the question as to whether it was due to release of the intestinal isoenzyme into the circulation. If this were the case it might form the basis of a test for acute intestinal ischaemia, which could be used in the clinical laboratory. Sera from three dogs were kindly examined for us by Dr M. Yong, of the Department of Chemical Pathology, St George's Hospital, London, by his agar electrophoretic technique. No preponderance of the intestinal isoenzyme was found in any of these specimens and the rise in alkaline phosphatase appeared almost certainly to be due to release of material from the liver. The marked difference noted between the major devascularization groups and the controls suggests that this is not related purely to the operative procedure, which itself inevitably involves some handling of the liver. A possible explanation is that it is a result of the portal bacteraemia or toxaemia occasioned by the colonic lesion, but without bacteriological examination of the portal 
blood this must remain a speculation. The prevalence of raised alkaline phosphatase levels in patients with ischaemia of the colon is being currently investigated.

The earliest change noted radiologically after colonic devascularization is 'thumb printing', and the suggestion of Boley et al (1963) that this appearance is due to mucosal oedema and haemorrhage has been confirmed by our sigmoidoscopic findings. Boley makes the further point that the disappearance of 'thumb printing' in later barium studies is a most important feature in the radiological diagnosis of vascular disease of the colon, and we entirely support this view. Ulceration of the mucosa which can be seen both sigmoidoscopically and on the pathological specimen is not demonstrated on the $x$-ray films in these experimental animals. The mature ischaemic lesion presents as a stricture on the barium film, which is constant following insufflation of air (Fig. 8), but in our series complete correlation of radiological and pathological stricturing was only found on one occasion.

Broadly speaking, the appearances found after deliberate devascularization of the colon in the experimental animal are similar to those seen in human ischaemic colitis (Marston et al, 1966; Payan, Levine, Bronstein, and King, 1965). There are of course differences, which are probably related to the comparative anatomy of the colonic microcirculation in the two species. Furthermore, the very early changes created and studied in the experimental laboratory do not often come the way of the clinical pathologist, who is usually presented with mature and florid lesions.

The pathology of ischaemic colitis in man has been described by McGovern and Goulston (1965) and by Marston et al (1966). The majority of the specimens studied by these authors showed focal strictures resulting from previous ischaemic episodes. The salient pathological features were stricture formation with mucosal ulceration, and widening of the submucosa, which was filled with granulation tissue containing haemosiderin-laden macrophages.

Extensive necrotic ulcers with full-thickness loss of mucosa and vascular granulation tissue replacing the submucosa are similar in the experimental and clinical material. The only significant difference is in the numbers of haemosiderin-laden macrophages, which are prominent in human material. They are not a major feature of the experimental lesions, though they are present in small numbers. Epithelial regeneration at the margins of the ulcers as previously noted (Marston et al, 1966) was not often seen, but all the ulcers examined were early lesions, from animals sacrificed at one or 14 days. The patchy atrophy of mucosa seen in the human material is very similar to the changes round the small discrete ulcers in the experimental specimens.

Although the normal dog's colon contains considerably more fibrous tissue than does that of man, the extent of fibrosis following ischaemia is greater in the human material than in these experimental specimens, and gross fibrosis of muscle with infiltration by vascular granulation tissue was only found in one preparation. Changes in the submucous arterioles seen in the clinical specimens were not found in the experimental material, but the peritoneal appearances were identical.

\section{SUMMARY}

Changes following interruption of the arterial supply to the colon have been studied in dogs, using three devascularizing operations of different extent and allowing the lesion to mature for varying periods of time.

This procedure is well tolerated by the healthy animal and only rarely causes a major systemic illness. Characteristic sigmoidoscopic appearances are described.

The earliest change seen radiologically was 'thumb printing' of the affected segment, due to mucosal oedema and haemorrhage, which usually reverted to normal within a few weeks, and was accompanied by a varying degree of spasm. Occasionally a persistent stenosis resulted.

A brisk peripheral leucocytosis was noted following operation in all cases, which was unrelated to the degree of vascular injury.

Postoperative serum levels of lactic dehydrogenase, glutamic oxalate transaminase, and glutamic pyruvate transaminase did not vary significantly from the preoperative baseline. The serum level of alkaline phosphatase rose sharply in the immediate postoperative period, and was directly related to the extent of devascularization and mucosal necrosis. The possible reasons for this are discussed.

The pathological appearances, depending on the degree of ischaemia inflicted, varied from slight mucosal congestion to extensive necrotic ulceration. On occasion a fibrous stricture resulted. The lesions are broadly similar to those seen in human ischaemic colitis, but differ from them in the smaller number of haemosiderin-laden macrophages and the lesser degree of fibrosis.

We wish to thank Professor R. H. S. Thompson and Dr A. L. Miller of the Courtauld Institute of Biochemistry for permission to use their facilities and for performing the serum enzyme estimations for us. The radiography was carried out by Sister M. V. Tomlinson of the Department of X-ray Diagnosis. The wellbeing of the animals depended greatly on the meticulous care 
given them by Mr A. C. Catchpole and his staff. Much invaluable help was received from $\mathrm{Mr}$ H. S. Drury, medical artist to the Hospital, and $\mathrm{Mr} \mathrm{M}$. Turney of the Department of Photography.

Part of this work was communicated to the Surgical Research Society at their meeting in Dublin in June 1966.

\section{REFERENCES}

Bedrak, E. (1965). Blood serum enzyme activity of dogs exposed to heat stress and muscular exercise. J. appl. Physiol., 20, 587-590.

Boley, S. J., Schwartz, S., Lash, J., and Sternhill, V. (1963). Reversible vascular occlusion of the colon. Surg. Gynec. Obstet., 116, 53-60.

_-, Krieger, H., Schultz, L., Robinson, K., Siew, F. P., Allen, A. C., and Schwartz, S. (1965). Experimental aspects of peripheral vascular occlusion of the intestine. Ibid., 121, 789-794.

Clayden, E. C. (1962). Practical Section Cutting and Staining, 4th ed. Churchill, London.

Dagher, F. J., Panossian, A., and Saab, S. (1967). The effect of experimental ligation of the superior mesenteric artery on serum xanthine oxidase and transaminase activity. Surgery, 62, 1044-1050.

Hukuhara, T., Kotani, S., and Sato, G. (1961). Effects of destruction of intramural ganglion cells on colonic motility: possible genesis of congenital megacolon. Jap. J. Physiol., 11, 635-640.

McGovern, V. J., and Goulston, S. J. M. (1965). Ischaemic enterocolitis. Gut, 6, 213-220.

Marston, A. (1962). Causes of death in mesenteric arterial occlusion. I. Local and general effects of devascularization of the bowel Ann. Surg., 158, 952-959.

- Pheils, M. T., Thomas, M. L., and Morson, B. C. (1966). Ischaemic colitis. Gut, 7, 1-15.

Miller, R. E., and Knox, W. G. (1966). Colon ischaemia following infrarenal aortic surgery. Ann. Surg., 163, 639-642.

Payan, H., Levine, S., Bronstein, L., and King, E. (1965). Subtotal ischemic infarction of colon simulating ulcerative colitis. Arch. Path., 80, 530-533.

Rudolph, L. A., Schaefer, J. A., Dutton, R. E., Jr, and Lyons, R. H. (1957). Serum glutamic oxalacetic transaminase in experimental tissue injury. J. clin. Lab. Invest., 49, 31-40.

Sturdy, D. E. (1968). Non-specific (ischaemic) segmental colitis. Brit. J. Surg., 55, 99-101.

de Villiers, R. (1966). Ischaemia of the colon: an experimenta study. Ibid., 53, 497-503. 